PROCEEDINGS OF THE

AMERICAN MATHEMATICAL SOCIETY

Volume 139, Number 2, February 2011, Pages 619-625

S 0002-9939(2010)10503-8

Article electronically published on September 17, 2010

\title{
UPPER-BOUND FOR THE NUMBER OF ROBUST PARABOLIC CURVES FOR A CLASS OF MAPS TANGENT TO IDENTITY
}

\author{
FRANCESCO DEGLI INNOCENTI AND CHIARA FROSINI
}

(Communicated by Franc Forstneric)

\begin{abstract}
In this paper we provide an upper-bound for the number of robust parabolic curves for germs of biholomorphisms in $\mathbb{C}^{2}$ which are tangent to the identity and which are time-one flows of a holomorphic vector field.
\end{abstract}

\section{INTRODUCTION}

The Leau-Fatou flower theorem 9 completely describes the dynamic behavior of 1-dimensional maps tangent to the identity. In dimension two Abate [1, Écalle [11] and Hakim [12] proved that if $f$ is a holomorphic map tangent to the identity in $\mathbb{C}^{2}$ and $\nu(f)$ is the degree of the first non-vanishing jet of $f-I d$, then there exist $\nu(f)-1$ robust parabolic curves (RP curves for short) for $f$, that is to say, attractive petals at the origin which survive under blow-up (see 3] and Section 2). A new approach in studying dynamics of germs of mappings tangent to the identity has been introduced in [2] and [6] by Abate, Bracci and Tovena. Their idea consists in studying discrete dynamics using families of vector fields whose flows are approximations at the first order of $f$. This technique turns out to be very useful.

In general, given a germ tangent to the identity there exist no holomorphic vector fields whose flows coincide with such a germ. The class of tangent to the identity germs which are time-one maps of holomorphic vector fields will be denoted by $\Phi_{\geq_{2}}\left(\mathbb{C}^{2}, 0\right)$. Such a class is dense in the space of germs tangent to the identity and the dynamical properties of elements of $\Phi_{\geq 2}\left(\mathbb{C}^{2}, 0\right)$ can be related rather directly to the dynamical properties of the associated vector fields. Indeed, it is in this class that we give a bound on the number of RP curves, showing that any RP curve for the germs belongs to a separatrix for the associated vector field and then exploiting a result by Corral and Fernandez-Sanchez [10] to estimate the number of separatrices.

Theorem 1.1. Let $f=\left(f_{1}, f_{2}\right) \in \Phi_{\geq 2}\left(\mathbb{C}^{2}, 0\right)$ be a non-dicritical holomorphic map. Set $\eta(f):=\max \left\{\operatorname{ord}\left(f_{1}-I d\right)\right.$, ord $\left.\left(f_{2}-I d\right)\right\}$ and let $\mu(f)$ be the Milnor number of $f$. Then the number of robust parabolic curves is at most

$$
(\mu(f)+1)^{2}\left(\eta^{2}(f)+\eta(f)\right) .
$$

Received by the editors August 31, 2009 and, in revised form, February 23, 2010 and March 15, 2010.

2010 Mathematics Subject Classification. Primary 32H50, 37F75, 32M25, 32S65.

(C)2010 American Mathematical Society

Reverts to public domain 28 years from publication 
In 6] Bracci proved that $f$ is dicritical if and only if it has infinitely many parabolic curves. Here we show that the parabolic curves at a dicritical point are indeed robust ones:

Proposition 1.2. Let $f=\left(f_{1}, f_{2}\right) \in \Phi_{\geq 2}\left(\mathbb{C}^{2}, 0\right)$ be a dicritical holomorphic map. Then there exist infinitely many robust parabolic curves.

It is worthwhile to notice that there is a well known classical result that links a holomorphic map tangent to the identity in $\mathbb{C}^{2}, f \in \Phi_{\geq 2}\left(\mathbb{C}^{2}, 0\right)$, with a holomorphic vector field, $X$, such that $\exp (X)=f$ (see e.g. 44):

Proposition 1.3. Let $X$ be a germ of holomorphic vector in $\left(\mathbb{C}^{2}, 0\right)$. Then its flow can be written as

$$
f^{t}(x, y)=\left(x+\sum_{n=1}^{\infty} \frac{t^{n}}{n !} X^{n} \cdot x, y+\sum_{n=1}^{\infty} \frac{t^{n}}{n !} X^{n} \cdot y\right)
$$

where $X^{n} \cdot x$ is defined as $X$ applied to $X^{n-1} \cdot x$ and $X . x$ is the application of $X$ to $x$.

Remark 1.4. If $f$ is a holomorphic map tangent to the identity in $\mathbb{C}^{2}$ at the origin, then the associated vector field has order of singularity at 0 greater than or equal to 2 .

For the sake of clarity we now briefly recall some useful definitions for vector fields and maps (see also [1, 6], 7]).

Definition 1.5. Let $X$ be a holomorphic vector field on an open neighborhood $p \in$ $U \subseteq \mathbb{C}^{2}$ and let $p$ be a singularity for $X$. The vector field $X$ defines a holomorphic foliation, $\mathcal{F}$, on $U$. Let blow-up $X$ at $p$ and consider the normalized pull-back foliation $\tilde{\mathcal{F}}$. If the exceptional divisor is not invariant for $\tilde{\mathcal{F}}$, we say that $p$ is a dicritical singularity.

Analogously it is possible to define dicritical singularity for holomorphic maps. To give such a definition we need some preliminary notation.

Let $S$ be an irreducible curve in $\mathbb{C}^{2}$ and let $f: \mathbb{C}^{2} \rightarrow \mathbb{C}^{2}, f \neq I d$, be a holomorphic map such that $\left.f\right|_{S}=I d_{S}$. Let $\mathcal{I}(S)_{p} \subset \mathcal{O}_{p}$ be the ideal of germs vanishing on $S$. Let $l$ be a defining function for $S$ at $p$. Then $f=I d+l^{T} G$ for some germ $G=\left(G_{1}, G_{2}\right)$ of holomorphic self map of $\mathbb{C}^{2}$ at $(0,0), G \not \equiv 0$ on $S$ and $T \geq 1$. We call $T$ the order of $f$ on $S$ at $p$.

Definition 1.6. We say that $f$ is tangential on $S$ at $p$ if

$$
\frac{l \circ f-l}{l^{T}} \equiv 0 \quad \bmod \mathcal{I}(S)_{p}
$$

In this setting we give the following definition:

Definition 1.7. Let $f$ be a holomorphic map tangent to the identity in $\mathbb{C}^{2}$. We say that 0 is dicritical for $f$ if the blow-up of $f$ is non-tangential on the exceptional divisor.

We would like to sincerely thank the referee for useful comments and remarks. 


\section{Robust PARABOlic CURVES}

The aim of this section is to introduce the definition of parabolic and robust parabolic curves for a germ of holomorphic maps tangent to the identity (at the origin in $\mathbb{C}^{2}$ ) and to study the existing relationship between the separatrices of the vector field associated to $f$ and these curves. For the sake of clearness we underline that in this paper we consider only (locally) irreducible separatrices. As a matter of notation let $\mathbb{D}$ be the unit complex disc in $\mathbb{C}$ and let $\Delta_{r}, r \in \mathbb{R}$, be the disc in $\mathbb{D}$ of center 0 and radius $r$. We will denote by $\pi:(M, D) \rightarrow\left(\mathbb{C}^{2}, 0\right)$ the blow-up of $\mathbb{C}^{2}$ at the origin, with $D=\pi^{-1}(0)=\mathbb{P}^{1}$. Moreover if $f$ is a holomorphic map tangent to the identity in $\mathbb{C}^{2}$ at the origin (in short $f \in \operatorname{Diff}\left(\mathbb{C}^{2}, 0\right)$ ), we will denote by $\tilde{f}$ the unique germ of diffeomorphism in $(M, D)$ such that $\pi \circ \tilde{f}=f \circ \pi$ and $\left.\tilde{f}\right|_{D}=\left.i d\right|_{D}$. Analogously, given $X$ a vector field, we will denote by $\bar{X}$ the vector field in $(M, D)$ such that $D \pi \cdot \bar{X}=X \circ \pi$.

Definition 2.1. A parabolic curve for a holomorphic map $f$ tangent to the identity at the origin in $\mathbb{C}^{2}$ is an injective holomorphic map $\varphi: \Delta \rightarrow \mathbb{C}^{2}$ satisfying the following properties:

i) $\Delta$ is a simply connected domain in $\mathbb{C}$ with $0 \in \partial \Delta$;

ii) $\varphi$ is continuous at the origin, and $\varphi(0)=0$;

iii) $\varphi(\Delta)$ is invariant under $f$, and $\left(\left.f\right|_{\varphi(\Delta)}\right)^{n} \rightarrow 0$ as $n \rightarrow \infty$.

As mentioned in the introduction the idea of a robust parabolic curve was first introduced by Abate and Tovena in [3]:

Definition 2.2. A robust parabolic curve $\varphi$ is a parabolic curve that satisfies the following conditions:

a) we can blow-up $\varphi$ at level $h$ for any $h \geq 1$,

b) there is a formal power series $Q \in(\overline{\mathbb{C}}[[x]])^{2}$ such that for every $h \geq 1$ there is $r_{h}>0$ such that $\varphi-Q_{h}=O\left(\zeta^{h+1}\right)$ in $\Delta_{r_{h}}$, where $Q_{h}$ denotes the truncation at degree $h$ of $Q$.

Remark 2.3. Essentially condition a) means that the strict transform of the parabolic curve is also a parabolic curve. For a clearer definition of "blow-up at level $h$ " we refer to 3 .

The importance of RP curves is expressed by the following theorem of Abate and Tovena (see Theorem 1.5 of [3]):

Theorem 2.4. Let $f \in \operatorname{Dif} f\left(\mathbb{C}^{2}, 0\right)$ be tangent at the identity, and assume that the origin is an isolated fixed point of $f$. Then $f$ admits at least one robust parabolic curve.

The geometric meaning of robust parabolic curve is clarified by the following proposition:

Proposition 2.5. Let $f \in \Phi_{\geq 2}\left(\mathbb{C}^{2}, 0\right)$ be a holomorphic map and let $X$ be a vector field such that $\exp (X)=f$. Let $\varphi$ be a robust parabolic curve. Then $\varphi$ is contained in a (locally) irreducible separatrix of $X$. Conversely in every (locally) irreducible separatrix of $X$ there exists at least one robust parabolic curve for $f$.

Proof. Consider $p \in \varphi(\Delta)$. Since

$$
\exp (X)=f
$$


the orbit $\left\{f^{n}(p)\right\}_{n \in \mathbb{N}}$ is contained in a (locally) irreducible separatrix $S$ of $X$. We want to prove that every orbit generated by any $q \in \varphi(\Delta)$ stays inside $S$. Assume by contradiction that we can find two orbits that converge to zero living in two different separatrices, say $S_{1}$ and $S_{2}$. Let $l_{1}(x, y)$ and $l_{2}(x, y)$ be, respectively, the local expressions of $S_{1}$ and $S_{2}$ and let $h$ be the order of the first non-zero jet of $l_{1}-l_{2}$. If we blow-up $h$ times the foliation associated to the vector field, then, by property a) of the definition of RP curves, the two orbits converge to zero with two different directions and this contradicts property b) of Definition 2.2 .

Let us prove the converse and let us divide the proof into two steps. First we assume that $S$ is a non-singular (locally) irreducible separatrix for the vector field $X$. Without loss of generality we can suppose that its analytic expression is $y=0$. Thus the vector field restricted to the (locally) irreducible separatrix $\{y=0\}$ is $\left(A(x, y) \frac{\partial}{\partial x}\right)$ and its exponential is tangent to the identity and given by $(x, y) \mapsto\left(x+x^{h}+\cdots, y\right)$. By Theorem 2.4 we get the assertion. We now proceed with the second step and consider the singular case; that is, we suppose that $S$ is a singular (locally) irreducible separatrix for $X$. In this case we solve the singularity by means of a finite number of blow-ups, and by Lemma 2.4 in 8 , we get a vector field $\bar{X}$ such that $\tilde{f}=\exp \bar{X}$. We proceed as in step one and we claim that, after blowing down, we get an RP curve, $\varphi=\left(\varphi_{1}, \varphi_{2}\right)$, for the map $f$.

Indeed $\varphi$ is definitely a parabolic curve and we can blow it up at level $h$ for any $h \geq 1$. Moreover let $\tilde{Q}=\left(\tilde{Q}_{1}, \tilde{Q}_{2}\right)$ be the formal power series such that $\tilde{\varphi}-\tilde{Q}_{h}=$ $O\left(\zeta^{h+1}\right)$ in $\Delta_{r_{h}}$, as in Definition $2.2(\mathrm{~b})$. Set $Q:=\pi(\tilde{Q})=\left(\tilde{Q}_{1}, \tilde{Q}_{1} \tilde{Q}_{2}\right)$. Then

$$
\varphi-Q=\left(\varphi_{1}-\tilde{Q}_{1}, \tilde{\varphi}_{1} \tilde{\varphi}_{2}-\tilde{Q}_{1} \tilde{Q}_{2}\right) .
$$

Let $\tilde{Q}_{i}^{h}, i=1,2$, denote the truncation at degree $h$. By an easy calculation we see that

$$
\begin{aligned}
\tilde{\varphi}_{1}(\zeta) \tilde{\varphi}_{2}(\zeta) & -\tilde{Q}_{1}(\zeta) \tilde{Q}_{2}(\zeta)=\tilde{\varphi}_{1}(\zeta) \tilde{\varphi}_{2}(\zeta)-\tilde{Q}_{1}^{h}(\zeta) \tilde{\varphi}_{2}(\zeta) \\
& +\tilde{Q_{1}^{h}}(\zeta) \tilde{\varphi}_{2}(\zeta)-\tilde{Q_{1}^{h}}(\zeta) \tilde{Q_{2}^{h}}(\zeta)+\tilde{Q_{1}^{h}}(\zeta) \tilde{Q}_{2}^{h}(\zeta)-\left[\tilde{Q}_{1}(\zeta) \tilde{Q}_{2}(\zeta)\right]^{h}=O(\zeta)^{h+1}
\end{aligned}
$$

Thus properties a) and b) of Definition 2.2 are fulfilled and the proposition is proved.

\section{NON-DiCRITICAL CASE}

In this section we prove Theorem 1.1]. In [10] Corral and Fernandez-Sanchez find the optimal estimate of the number of separatrices by the Milnor number of $X$ (see [5] for the definition):

Proposition 3.1. Let $X$ be a holomorphic vector field in $\mathbb{C}^{2}$, singular at the origin. Let $S$ be the curve determined by all locally irreducible separatrices passing through the origin. Let $r_{0}(S)$ be the number of the irreducible components of $S$ and let $n_{0}(S)$ be the minimal number of blowing-ups needed to desingularize $S$. Then

$$
\begin{aligned}
& r_{0}(S) \leq \mu_{0}(X)+1, \\
& n_{0}(S) \leq \mu_{0}(X)+1,
\end{aligned}
$$

where $\mu_{0}$ is the Milnor number of $X$ at the origin.

In order to express the previous estimate in terms of invariants of $f$ we introduce the intersection multiplicity (see [1]). 
Proposition 3.2. Let $f \in \Phi_{\geq 2}\left(\mathbb{C}^{2}, 0\right)$ be a holomorphic map tangent to the identity in $\mathbb{C}^{2}$ and let $X$ be the associated vector field. Then the Milnor number of $X$ at the origin is equal to the intersection multiplicity of $f-I d$.

Proof. It suffices to observe that if

$$
X=A(x, y) \frac{\partial}{\partial x}+B(x, y) \frac{\partial}{\partial y},
$$

then by equation (11) the order of zero for $A, B$ and $f-I d$ is the same.

Proposition 2.5 shows that the RP curves live inside separatrices of the associated vector field. So the idea is to estimate the number of separatrices of the vector field and then the number of RP curves inside a separatrix.

Proof of Theorem 1.1. We start by estimating the number of RP curves that are in a separatrix. We divide the proof into two steps.

1) If $S$ is non-singular, we can proceed as in the first part of the proof of Proposition 2.5 and conjugate the restriction of $f$ to a map of the kind $(u, v) \mapsto\left(u+u^{h}+\cdots, v\right)$. Then we estimate the exponent $h$. An easy computation shows that the exponent $h$ is the lowest degree of the expression of $A(u, \psi(u))$. The same computation proves that the order of $A(u, v)$ is the same as the order $\nu_{1}$ of $f_{1}-I d$. Then

$$
A_{\nu_{1}}(u, \psi(u))=\sum_{i+j=\nu_{1}} u^{i} \psi(u)^{j}
$$

so the lowest degree is

$$
i+p j \leq p i+p j=\nu_{1} p
$$

for $0 \leq i, j \leq \nu_{1}$.

Then the number of RP curves in $S$ is estimated from above by $\max \left\{\nu_{1}, \nu_{2}\right\} p$.

This estimate depends on $p$ and then on the particular separatrix. It is possible to improve this result by removing the dependence on the separatrix in the following way. Let $y=\varphi(x)=x^{k}+\cdots$ be a separatrix so that

$$
\frac{d y}{d x}=\frac{B(x, y)}{A(x, y)} .
$$

Comparing the lowest degree of the above expressions we obtain that

$$
k=\nu_{2}-\nu_{1}+1 \leq \nu_{2}+1 \leq \max \left\{\nu_{1}, \nu_{2}\right\}+1=\eta(f)+1 .
$$

Thus we have $\eta(f)(\eta(f)+1)$ RP curves for every separatrix. Let us notice that in this case $\mu(f)=0$.

2) If the separatrix $S$ is singular, then, again, we solve the singularity, and by Proposition 3.1 the field $\bar{X}$ has at most $(\mu(f)+1)^{2}$ smooth separatrices. We then apply step one to each of these separatrices and get that the maximal number of RP curves is $(\mu(f)+1)^{2}\left(\eta^{2}+\eta\right)$. This completes the proof of Theorem 1.1. 


\section{DicRitical CASE}

The goal of this section is to estimate the number of RP curves in the dicritical case. From the theorem on existence and uniqueness of solutions of ordinary differential equations stated in [13] we have:

Proposition 4.1. Let $X$ be a holomorphic vector field and let $p$ be a dicritical singularity. Then there exist infinitely many locally irreducible separatrices through $p$.

In the discrete case the following result holds (see [1, [6]):

Proposition 4.2. Let $f$ be a map tangent to the identity in $\mathbb{C}^{2}$. If 0 is dicritical, then there exist infinitely many parabolic curves for $f$.

The last two propositions suggest that there exists a strict relationship between the two notions of dicriticity, namely:

Proposition 4.3. Let $f \in \Phi_{\geq_{2}}\left(\mathbb{C}^{2}, 0\right)$ be a map tangent to the identity in $\mathbb{C}^{2}$ and let $X$ be the vector field such that $\exp (X)=f$. Then $f$ is dicritical in 0 if and only if $X$ is dicritical in 0.

Proof. If $X$ is dicritical, then by Proposition 4.1 there exist infinitely many separatrices, and then, by Proposition 2.5, $f$ admits infinitely many RP curves. So $f$, by Theorem 1.1, has to be dicritical. To prove the converse, if $X$ were not dicritical, it would have only a finite number of separatrices and all the RP curves for $f$ would be contained therein. This implies that there are only a finite number of robust parabolic curves for $f$. Then $f$ is not dicritical, for otherwise it would have infinitely many RP curves. Indeed parabolic curves obtained as in 11 are infinite (see [6]), and moreover they are robust by Theorem 2.6 in 3 since they are obtained by the procedure in [6].

In this setting we have

Proposition 4.4. Let $f \in \Phi_{\geq 2}\left(\mathbb{C}^{2}, 0\right)$ be a dicritical holomorphic map tangent to the identity in $\mathbb{C}^{2}$. Then there exist infinitely many robust parabolic curves.

Proof. Since $f$ is dicritical, by Proposition 4.3, $X$ is dicritical. By Proposition 4.1 there exist infinite separatrices, and then by Proposition 2.5 we get the assertion.

\section{REFERENCES}

[1] M. Abate, The residual index and the dynamics of holomorphic maps tangent to the identity, Duke Math. J., 107 (2001), 173-207; MR.1815255 (2003a:32028)

[2] M. Abate, F. Bracci and F. Tovena, Index theorems for holomorphic self maps, Ann. of Math. (2), 159 (2004), 819-864; MR2081441 (2005g:32044)

[3] M. Abate, F. Tovena, Parabolic curves in $\mathbb{C}^{3}$, Abstr. Appl. Anal., 5 (2003), 275-294; MR.1981266 (2004c:32035)

[4] Abraham-Marsed-Ratiu, Tensor analysis and applications, Springer Applied Math. Sciences.

[5] W. Barth, C. Peters, A. Van de Ven, Compact complex surfaces, Springer Verlag (1984); MR749574 (86c:32026)

[6] F. Bracci, The dynamics of holomorphic maps near curves of fixed points, Ann. Scuola Norm. Sup. Pisa Cl. Sci., 5 (2003), 493-520; MR2020858 (2004i:32032)

[7] F. Bracci, F. Tovena, Residual indices of holomorphic maps relative to singular curves of fixed points on surfaces, Math. Z., 242 (2002), 481-490; MR1985461 (2004d:32018) 
[8] F. E. Brochero Martinez, F. Cano, L. Lopez Hernanz, Parabolic curves for diffeomorphisms in $\mathbb{C}^{2}$ Publ. Math., 52 (2008), 189-194; MR2384846 (2009a:37090)

[9] L. Carleson, T.W. Gamelin, Complex Dynamics, Springer (1992); MR1230383 (94h:30033)

[10] N. Corral, P. Fernandez-Sanchez, Isolated invariant curves of a foliation, Proc. Amer. Math. Soc., 134 (2006), 1125-1132; MR2196047 (2007b:32049)

[11] J. Écalle, Iteration and analytic classification of local diffeomorphisms of $\mathbb{C}^{n}$, Iteration theory and its functional equations, Lect. Notes in Math., 1163, Springer-Verlag, Berlin (1985), 4148; MR829756 (87g:58060)

[12] M. Hakim, Analytic transformations of $\left(\mathbb{C}^{p}, 0\right)$ tangent to the identity, Duke Math. J., 92 (1998), 403-428; MR1612730 (99a:32036)

[13] A. Seidenberg, Reduction of singularities of the differential equation Ady $=B d x$, Amer. J. Math., 90 (1968), 248-269; MR0220710 (36:3762)

Dipartimento di Matematica, Universitì di Pisa, Largo Bruno Pontecorvo 5, 56127, Pisa, ITALy

E-mail address: degliinno@dm.unipi.it

Dipartimento di Matematica, Università di Firenze, viale Morgagni 67/A, 50134, Firenze, Italy

E-mail address: frosini@math.unifi.it

Current address: Dipartimento di Ingegneria dell'Informazione, Universitá di Siena, Palazzo S. Niccolò, Via Roma 56, 53100, Siena, Italy

E-mail address: frosinichiara@dii.unisi.it 